\title{
Solution of Blasius Equation by Variational Iteration
}

\author{
Yucheng Liu ${ }^{1, *}$, Sree N. Kurra ${ }^{2}$ \\ ${ }^{1}$ Department of Mechanical Engineering, University of Louisiana, Lafayette, LA 70504, USA \\ ${ }^{2}$ The Center for Advanced Computer Studies, University of Louisiana, Lafayette, LA 70504, USA
}

\begin{abstract}
TheBlasius equation is a well known third-order nonlinear ordinary differential equation, which arises in certain boundary layer problems in the fluid dynamics. This paper presents a way of applying He's variational iteration method to solve the Blasius equation. Approximate analytical solution is derived and compared to the results obtained from Adomian decomposition method. Comparisons show that the present method is accurate and the using of He's method does accelerate the convergence of the power series. A robust and efficient algorithm is also programmed using Matlab based on the present approach, which can be easily employed to solve Blasius equation problems
\end{abstract}

Keywords Blasius Equation, He's Variational Iteration Method, Nonlinear Ordinary Differential Equation, Matlab

\section{Introduction}

In this paper, He's variational iteration method is employed to solve the Blasius equation. This method was developed by the Chinese mathematician Ji-Huan $\mathrm{He}$ as a modification of a general Lagrange multiplier method. The Blasius equation, the laminar viscous flow of fluid over a flat plate, is the vital equation for fluid dynamics and is represented by the following equation:

$$
\begin{aligned}
& f^{(3)}(x)+\frac{1}{2} f(x) \cdot f^{(2)}(x)=0, \\
& f^{(1)}(0)=f(0)=0, f^{(1)}(+\infty)=1
\end{aligned}
$$

Where $f(x)$ is the freestream velocity function, and ${ }^{(\mathrm{n})}$ denotes the nth order derivative.

The fluid problems governed by the Blasius equation have been solved by various numerical and analytical methods. Wang, Hashim, and Abbasbandy[1, 3] solved for the Blasius equation using the Adomian decomposition method and proved it to be accurate and reliable. $\mathrm{Yu}$ and Chen[4] employed the differential transformation method for solution and obtained not only the numerical values, but also the power series close-form solutions. Lin[5] used the parameter iteration method and compares the results with Howarth's numerical solution to solve for the Blasius equation. Yao and Chen[6] have solved the shrinking plate with constant velocity problem by using homotopy analysis techniques. Lastly, Parand and Taghavi[7] used a collocation method based on a rational scaled generalized Laguerre function collocation method to solve this equation.

He's variational iteration method (VIM) has been exten

* Corresponding author:

yucheng.liu@louisiana.edu (Yucheng Liu)

Published online at http://journal.sapub.org/am

Copyright (C) 2011 Scientific \& Academic Publishing. All Rights Reserved sively applied as a powerful tool for solving various kinds of problems[8-11]. Liu and Gurram[12] have solved for free vibration problems involving an Euler-Bernoulli beam using this method and obtained accurate results which were the same as with the Adomian decomposition method. Słota[13] obtained results for the heat equation using this method and verifies the accuracy by obtaining the exact solution. This method can be used to solve nonlinear equations in heat transfer[14], nonlinear Jaulent-Miodek equations[15], the Fokker-Planck equation[16],nonlinear equations with Riemann-Liouville's fractional derivatives[17], and the semi-linear inverse parabolic equation[18],etc. The advantages of using He's VIM for numerical problems were also confirmed from the previous works.

Besides the He's method, VIM has been extended for applications to systems of nonlinear ordinary differential equations (ODE) and chaotic systems. Batiha et al.[19] implemented the multistage VIM to solve a class of nonlinear system of first-order ODEs. Through their study, the domain of validity of the solutions via the standard VIM was extended by the simple multistage strategy. Goh et al.[20, 21] illustrated the strength of VIM in numerically solving the chaotic Rossler system, a three-dimensional system of ODEs with quadratic nonlinearities, by implementing the multistage VIM.

In this paper the authors will convert the Blasius equation into a linear and a non-linear term. A correction factor and a Lagrangian multiplier will be utilized in a Maclaurin series approximation. Stationary and boundary conditions define the parameters of each problem. Through this approximation technique, a rapidly convergent series of solutions will be obtained and proven to be simple, accurate, and effective.

\section{He's Variational Iteration Method}




\section{(VIM)}

In this section, the concept of He's variational iteration method is briefly introduced. Consider the general nonlinear differential equation given in the form

$$
\mathrm{Lu}(\mathrm{t})+\mathrm{Nu}(\mathrm{t})=\mathrm{g}(\mathrm{t})
$$

where $\mathrm{L}$ is a linear operator, $\mathrm{N}$ is a nonlinear operator, and $\mathrm{g}(\mathrm{t})$ is a known function. By using the variational iteration method, a correction functional can be constructed as

$$
u_{n+1}(t)=u_{n}(t)+\int_{0}^{t} \lambda\left(L u_{n}(\xi)+N \widetilde{u}_{n}(\xi)-g(\xi)\right) d \xi
$$

where $\lambda$ is a general Lagrange multiplier, which can be determined optimally via variational theory; the subscript $n$ means the $n$th approximation; $u_{n}$ is a restricted variation and $\delta u_{n}=0$.

\section{Using the He's VIM to Solve the Blasius Equation}

To solve the Blasiusequation, Eqn. (1) needs to be converted into the He's VIM form (Eqn. (2)) so that we have

$$
N u(t)=f^{(3)}(x)+\frac{1}{2} f(x) \cdot f^{(2)}(x) \text { and } \mathrm{g}(\mathrm{t})=0
$$

Eqn. (1) then can be transformed as

$$
f^{(3)}(t)+\frac{1}{2} f(t) \cdot f^{(2)}(t)=0
$$

With the correction function

$$
f_{n+1}(x)=f_{n}(x)+\int_{0}^{x} \lambda\left[f_{n}^{(3)}(t)+\frac{1}{2} f_{n}(t) \cdot f_{n}^{(2)}(t)\right] d t
$$

where $\lambda$ is the Lagrange multiplier.

$$
Y_{n+1}(x)=Y_{n}(x)+\int_{0}^{x} \lambda\left[Y_{n}^{(3)}(t)+\frac{1}{2} Y_{n}(t) \cdot Y_{n}^{(2)}(t)\right] d t
$$

Integrating the integral by parts and Eqn. (7) becomes

$$
\begin{aligned}
& Y_{n+1}(x)=Y_{n}(x)+\frac{1}{2} \lambda Y_{n}^{(2)}(x)-\frac{1}{2} \lambda^{(1)} Y_{n}^{(1)}(x)+\frac{1}{2} \lambda^{(2)} Y_{n}(x) \\
& +\frac{1}{2} \lambda Y_{n}(x) Y_{n}^{(1)}(x)-\frac{1}{2} \int_{0}^{x} \lambda^{(1)} Y_{n}^{(1)}(t) Y_{n}(t) d t-\frac{1}{2} \int_{0}^{x} \lambda\left[Y_{n}^{(1)}(t)\right]^{2} d t
\end{aligned}
$$

By taking the variation on both sides of above equation with respect to $\mathrm{Yn}$, we can obtain

$$
\begin{aligned}
& \delta Y_{n+1}(x)=\delta Y_{n}(x)+\frac{1}{2} \lambda \delta Y_{n}^{(2)}(x)-\frac{1}{2} \lambda^{(1)} \delta Y_{n}^{(1)}(x)+\frac{1}{2} \lambda^{(2)} \delta Y_{n}(x) \\
& +\frac{1}{2} \lambda \delta Y_{n}(x) Y_{n}^{(1)}(x)-\frac{1}{2} \int_{0}^{x} \lambda^{(1)} \delta Y_{n}^{(1)}(t) Y_{n}(t) d t-\frac{1}{2} \int_{0}^{x} \lambda\left[\delta Y_{n}^{(1)}(t)\right]^{2} d t
\end{aligned}
$$

The stationary conditions obtained from last equation are

$$
\begin{gathered}
1+\left.\lambda^{(2)}\right|_{t=x}=0 \rightarrow \lambda^{(2)}=-1 \\
\left.\lambda^{(1)}\right|_{t=x}=0 \rightarrow \lambda^{(1)}=0 \\
\left.\lambda\right|_{t=x}=0 \rightarrow \lambda=0
\end{gathered}
$$

From above equations, the Lagrange multiplier $\lambda$ can be derived as

$$
\lambda=-(t-x)^{2} / 2
$$

Next, by substituting Eqn.(10) into Eqn.(7), the correction function can be expressed as

$$
Y_{n+1}(x)=Y_{n}(x)-\int_{0}^{x} \frac{(t-x)^{2}}{2}\left[Y_{n}^{(3)}(t)+\frac{1}{2} Y_{n}(t) \cdot Y_{n}^{(2)}(t)\right] d t
$$

In order to start iteration using Eqn.(11), $\mathrm{Y}_{0}(\mathrm{x})$ is needed, which is represented Maclaurin series with the first three terms

$$
Y_{0}(x)=\sum_{m=0}^{2} \frac{x^{m}}{m !} Y^{(m)}(0)=Y(0)+Y^{(1)}(0) x+Y^{(2)}(0) \frac{x^{2}}{2}
$$

By substituting the given boundary conditions in Eqn. (1) and assuming $\mathrm{Y}^{(2)}(0)=0.332$ based on the conclusions of Howarth[22], following equation can be obtained from Eqn. (12)

$$
\mathrm{Y}_{0}(x)=0.166 x^{2}
$$

From Eqn.f(11) with $\mathrm{n}=0$, we have

$$
Y_{1}(x)=Y_{0}(x)-\int_{0}^{x} \frac{(t-x)^{2}}{2}\left[Y_{0}^{(3)}(t)+\frac{1}{2} Y_{0}(t) \cdot Y_{0}^{(2)}(t)\right] d t
$$

By substituting Eqn. (13) into Eqn. (14) we can get

$$
Y_{1}(x)=0.166 x^{2}-\int_{0}^{x} \frac{(t-x)^{2}}{2}\left[Y_{0}^{(3)}(t)+\frac{1}{2} Y_{0}(t) \cdot Y_{0}^{(2)}(t)\right] d t
$$

From Eqn. (13) we obtain $\mathrm{Y} 0(\mathrm{t})=0.166 \mathrm{t} 2, \mathrm{Y} 0(1)(\mathrm{t})=$ $0.332 \mathrm{t}, \mathrm{YO}(2)(\mathrm{t})=0.332$, and $\mathrm{Y} 0(3)(\mathrm{t})=0$. Substituting above values into Eqn. (15) and from the first iteration we can find

$$
\begin{aligned}
& Y_{1}(x)=0.166 x^{2}-\int_{0}^{x} \frac{(t-x)^{2}}{2}\left(0.166 \times 0.332 \times t^{2}\right) d t \\
& =0.166 x^{2}-0.0138\left(\frac{8 x^{5}}{15}-\frac{x^{6}}{2}\right)
\end{aligned}
$$

Here $\mathrm{Y} 1(\mathrm{x})=\mathrm{f}(\mathrm{x})$ because $\mathrm{Y} 1(\mathrm{x})$ is considered as the base function for all iterations, Eqn. (16) can be rewritten as

$$
\begin{aligned}
& f(x)=0.166 x^{2}-\int_{0}^{x} \frac{(t-x)^{2}}{2}\left(0.166 \times 0.332 \times t^{2}\right) d t \\
& =0.166 x^{2}-0.0138\left(\frac{8 x^{5}}{15}-\frac{x^{6}}{2}\right)
\end{aligned}
$$

Diferentiate Eqn. (17) with respect to $\mathrm{x}$ and one can have

$$
\begin{aligned}
& f^{(1)}(x)=0.332 x-0.0138\left(\frac{8 x^{4}}{3}-3 x^{5}\right) \\
& f^{(2)}(x)=0.332-0.0138\left(\frac{32 x^{4}}{3}-15 x^{4}\right)
\end{aligned}
$$

So that $\mathrm{f}(2)(0)=0.332=\alpha 1$.

Following above equations, the Blasius equation is fully solved and the numerical results are obtained by Matlab as shown in Table 1 for the first four iterations. The results are compared to those obtained by Abbasbandy[2]. The results and comparisons listed in that table show that a good approximation and quick convergence to the actual solution is 
achieved, thus the accuracy and efficiency of He's variational iteration method is validated.

Table 1. Results of Blasius equation obtained from He's VIM and ADM ( $\mathrm{k}$ is the number of iterations).

\begin{tabular}{|c|c|c|c|c|c|}
\hline \multicolumn{2}{|c|}{ Adomian Decomposition Method } & \multicolumn{3}{|c|}{ He's Variational Iteration Method } \\
\hline $\mathrm{k}$ & $\alpha_{\mathrm{k}}$ & $\begin{array}{c}\text { Accuracy } \\
(\%)\end{array}$ & $\mathrm{k}$ & $\alpha_{\mathrm{k}}$ & $\begin{array}{c}\text { Accuracy } \\
(\%)\end{array}$ \\
\hline 1 & 0.333333 & 0.384 & 1 & 0.332164 & 0.398 \\
\hline 2 & 0.336355 & 1.294 & 2 & 0.334612 & 1.146 \\
\hline 3 & 0.336422 & 1.315 & 3 & 0.334655 & 1.421 \\
\hline 4 & 0.336423 & 1.315 & 4 & 0.334656 & 1.421 \\
\hline
\end{tabular}

\section{Conclusions}

In this paper, the Blasius equation is successfully solved using He's variational iteration method though a Matlab program. As shown by the results and comparisons listed in Table 1, the use of He's method to obtain solutions to the Blasius equation problems is weighed against the Adomian decomposition method. The number of calculations required for this method is lower than ADM yet it provides comparable accuracy and reliability. By using He's method, each iteration gives a direct approximation to the solution; whereas, by using $\mathrm{ADM}$, each iteration only gives the components of the solution and since the number of iterations needed for the required accuracy is not known beforehand, those components have to be summed continually after each iteration in order to obtain the full, approximate solution. Moreover, using ADM to solve for the nonlinear differential equations does require the generation of Adomian polynomials. As the number of iterations increases, the amount of calculations needed to obtain the new Adomian polynomial also increases and the process of obtaining these polynomials becomes tedious and time consuming. Such drawback is eliminated through the application of He's variational iteration method, which does not require the additional computations of the Adomian polynomials. In other words, compare to ADM, He's method is a more robust, accurate, and speedy way for solving the nonlinear differential equations.

In the future, He's method could be extensively applied for solving more nonlinear differential equations such as Poisson's equation, which is broadly used in electrostatics and mechanical engineering problems to define the Gaussian charge density of a given charge distribution. Meanwhile, the method presented in this paper could also be used to solve for the Lorenz attractor problem. The Lorenz equations are important nonlinear ordinary differential equations which are practically used in climate prediction of convection rolls in the atmosphere, laser design, and dynamo design. Moreover, the presented approach can also be applied to any heat transfer and flow problem leading to a coupled nonlinear system of ODEs, such as the steady Navier-Stokes equations, which are used to model the magnetohydrodynamic (MHD) flow of a non-Newtonian fluid in the presence of thermal radiation. Thus, the present method and the approach illustrated in this paper have broad applicability in engineering practices.

\section{Appendix - Matlab Code for the Pre- sented Algorithm}

syms b t a;

$\mathrm{y}(1)=0.332 * \mathrm{a}^{\wedge} 2 / 2$;

fori $=1: 5$

$y a(i)=\operatorname{subs}(y(i), a, t)$;

$y b(i)=\operatorname{subs}(y(i), a, p)$;

$y(i+1)=$

y(i) $1 / 2 * \operatorname{int}\left((\mathrm{ta})^{\wedge} 2 *\left(\operatorname{diff}(y a(\mathrm{i}), \mathrm{t}, 3)+\mathrm{b}^{*} \mathrm{ya}(\mathrm{i}) * \operatorname{diff}(\mathrm{ya}(\mathrm{i}), \mathrm{t}, 2)\right), \mathrm{t}, 0\right.$, a);

end

sol= $\operatorname{diff}(y(i+1), a, 2)$;

$\mathrm{p}=\operatorname{subs}($ sol, $a, 0)$;

end

\section{REFERENCES}

[1] L. Wang. "A new algorithm for solving classical Blasius equation", Applied Mathematics and Computation, 157(1), 2004, 1-9.

[2] I. Hashim. "Comments on 'A new algorithm for solving classical Blasius equation' by L. Wang”, Applied Mathematics and Computation, 176(2), 2006, 700-703.

[3] S. Abbasbandy "A numerical solution of Blasius equation by Adomian's decomposition method and comparison with homotopy perturbation method", Chaos, Solitons and Fractals, $31,2007,257-260$.

[4] L. Yu, C. Chen. "The solution of the Blasius equation by differential transformation method", Mathematical and Computer Modeling, 28(1), 1998, 101-111.

[5] J. Lin. "A new approximate iteration solution of Blasius equation", Communications in Nonlinear Science and $\mathrm{Nu}-$ merical Simulation, 4(2), 1999, 91-94.

[6] B. Yao, J. Chen. "A new analytical solution branch for the Blasius equation with a shrinking sheet", Applied Mathematics and Computation, 215(3), 2009, 1146-1153.

[7] K. Parand, A. Taghavi. "Rational scaled generalized Laguerre function collocation method for solving the Blasius equation", Journal of Computational and Applied Mathematics, 233(4), 2009, 980-989.

[8] J.-H. He, "Variational iteration method - a kind of non-linear analytical technique: some examples", International Journal of Non-Linear Mechanics, 34(4), 1999, 699-708.

[9] J.-H. He, "Variational iteration method for autonomous ordinary differential systems", Applied Mathematics and Computation, 114(2/3), 2000, 115-123.

[10] J.-H. He, "Variational iteration method for delay differential equations", Communications in Nonlinear Science and $\mathrm{Nu}-$ merical Simulation, 2(4), 1997, 235-236. 
[11] S.-Q. Wang, J.-H. He, "Variational iteration method for solving integro-differential equations", Physics Letters A, 367(3), 2007, 188-191.

[12] Y. Liu, C.S. Gurram, "The use of He's variational iteration method for obtaining the free vibration of an Euler-Bernoulli beam", Mathematical and Computer Modelling, 50(11/12), $2009,1545-1552$.

[13] D. Słota. "Exact solution of the heat equation with boundary condition of the fourth kind by He's variational iteration method", Computers \& Mathematics with Applications, 58(11/12), 2009, 2495-2503.

[14] H. Taria, D.D. Ganji, H. Babazadeh. "The application of He's variational iteration method to nonlinear equations arising in heat transfer", Physics Letters A, 363(3), 2007, 213-217.

[15] D.D. Ganji, M. Jannatabadi, E. Mohseni, “Application of he's variational iteration method to nonlinear Jaulent-Miodek equations and comparing it with ADM", Journal of Computational and Applied Mathematics, 207(1), 2007, 35-45.

[16] M. Dehghan, M. Tatari, "The use of He's variational iteration method for solving a Fokker-Planck equation", PhysicaScripta, 74, 2006, 310-316.

[17] S. Abbasbandy, "An approximation solution of a nonlinear equation with Riemann-Liouville's fractional derivatives by He's variational iteration method", Journal of Computational and Applied Mathematics, 207(1), 2007, 53-58.

[18] S.M. Varedi, M.J. Hosseini, M. Rahimi, D.D. Ganji, "He's variational iteration method for solving a semi-linear inverse parabolic equation", Physics Letters A, 370(3/4), 2007, 275-280.

[19] B. Batiha, M.S.M. Noorani, I. Hashim, E.S. Ismail, "The multistage variational iteration method for a class of nonlinear system of ODEs", PhysicaScripta, 76(4), 2007, 388-392.

[20] S.M. Goh, M.S.M. Noorani, I. Hashim, "A new application of variational iteration method for the chaotic Rossler system", Chaos, Solitons and Fractals, 42(3), 2009, 1604-1610.

[21] S.M. Goh, M.S.M. Noorani, I. Hashim, M.M. Al-Sawalha, "Variational iteration method as a reliable treatment for the hyperchaoticRossler system", International Journal of Nonlinear Sciences and Numerical Simulation, 10(3), 2009, 363-371.

[22] L. Howarth, "On the solution of the laminar boundary layer equations", Proceedings of The Royal Society A: Mathematical, Physical \& Engineering Sciences, 164, 1938, 547-579. 